Trans. JSASS Space Tech. Japan

Vol. 7, pp. 1-10, 2009

\title{
The Role of Vortices in Side Jet/Blunt Body Interaction at Hypersonic Speed
}

\author{
By Mohammed K. IBRAHIM, ${ }^{1,2)}$ Tetsuya NAKAMURA, ${ }^{2), *}$ Keiichi KITAMURA, ${ }^{2),+}$ \\ Koichi $\mathrm{MORI}^{2)}$ and Yoshiaki NAKAMURA ${ }^{2)}$ \\ 1) Aerospace Engineering Department, Faculty of Engineering, University of Cairo, Giza, Egypt \\ 2) Aerospace Engineering Department, Graduate School of Engineering, Nagoya University, Nagoya, Japan
}

(Received May 12th, 2008)

\begin{abstract}
A numerical investigation was carried out for a blunt body with a circular sonic air jet normally injected into a hypersonic flow from the body surface, where the angle of attack was changed up to $40^{\circ}$. Computed results were compared with the experimental data collected using the shock tunnel of Nagoya University. The aerodynamic interaction due to the jet creates complicated flow fields, which are rather difficult to analyze alone experimentally. The computed results show good agreement with the experimental data with regard to surface pressure distribution and schlieren visualization. It was found that at rather low angles of attack, two vortices, i.e., a separation vortex and a horseshoe vortex, are formed inside the separated boundary layer upstream of the jet. The location of these vortices corresponds to low-pressure regions in the pressure distribution. On the other hand, at a rather high angle of attack, the interaction produces a complex flow field, where vortices have little influence on the pressure distribution. Finally the jet interaction was found to enhance the jet reaction forces by about $35 \%$ - $45 \%$ on the body surface, the value of which is close to the experimental data.
\end{abstract}

Key Words: Hypersonic Flow, Side Jet, Aerodynamic Interaction, CFD

\section{Nomenclature}

$\begin{array}{ll}A & : \text { cross-sectional area } \\ F & : \text { force } \\ K & : \text { interaction amplification factor } \\ L & : \text { model length } \\ R & : \text { gas constant } \\ R e_{L} & : \text { Reynolds number based on model } \\ V & \text { length } \\ x, y, z & : \text { Clow velocity } \\ \delta_{b} & : \text { blunted cone half-angle } \\ \delta_{e} & : \text { external potential angle at the edge of } \\ & \text { the boundary layer } \\ \delta_{s} & : \text { skin-friction lines angle with body axis } \\ \gamma & : \text { specific heat ratio } \\ \theta & : \text { meridian angle }(\theta=0 \text { on the leeward } \\ \Upsilon & \text { side on the symmetry plane } \\ \text { Subscripts } & : \text { thrust } \\ b & : \text { model base } \\ j & : \text { case without jet injection } \\ J-O F F & : \text { case with jet injection } \\ J-O N & : \text { stagnation condition } \\ O & : \text { condition in vacuum } \\ V A & : \text { free-stream condition } \\ \infty & \end{array}$

\section{Introduction}

Conventional control surfaces are difficult for controlling the maneuvers of a flight vehicle under the situation of a low-density atmosphere or considerable aerodynamic heating at hypersonic speeds. The lateral jet control of a re-entry vehicle has an advantage over conventional control surfaces in that it does not affect the external aerodynamics of the flight vehicle except during the jet-activated period. ${ }^{1)}$ Even in the situation where interaction forces become very small, as in rarefied flows, jet thrust itself is still available for control. The flow field that results from the interaction between the jet and the external flow is complicated, the current understanding of which relies heavily on experimental data. ${ }^{2)}$

The concept of high-speed flight vehicles equipped with a side jet to control their attitude has been investigated since the $1960 \mathrm{~s}$, where many researchers have examined the flat plate problem ${ }^{3)}$ or the slender body problem ${ }^{2,4)}$ with a side jet in high-speed flows at low attack angles. Thus, many characteristic features have been clarified with regard to the aerodynamic interaction due to the side jet. ${ }^{5,6)}$ In addition, several analytical models to explain those features have been proposed. ${ }^{7)}$ However, the characteristics of blunted bodies with side jet control and 
the effects of the angle of attack on aerodynamic interaction are limited in literature and rely on experiments. ${ }^{8,9,10,11)}$ Kurita et al. ${ }^{9)}$ conducted extensive experimental investigations to explore the side jet interaction in a supersonic flow. The surface oil flow visualization in their investigation provided a good understanding of the flow structure with interaction. Their work was extended to hypersonic flow ${ }^{10,11)}$ using the shock tunnel at Nagoya University. As this shock tunnel has a testing duration of about 50 milliseconds, it is difficult to perform detailed investigations including surface oil flow visualization to examine properties of the interaction.

Oil streak lines in the oil flow visualization technique are the same as the skin friction lines (i.e., the loci of the local skin friction vectors). Skin friction patterns containing swept lines of attachment, separation, and reattachment, in association with a limited number of singular points (nodes and saddles) constitute the skeleton structure around which the elements of the entire flow field can be assembled. ${ }^{12)}$

It should be noted that skin friction patterns can be easily misinterpreted. Moreover, it is difficult to construct the correct external flow field from surface flow patterns.

In the meantime, numerical simulation can produce extensive and detailed flow information that cannot be provided experimentally. Therefore, it is indispensable to investigate this complex aerodynamic interaction problem using computational fluid dynamics (CFD). However, even in this case, experimental data are still useful to compare simulated results.

The main objective of the present work is to quantitively and qualitatively clarify the mechanism of jetinteracted flow fields at a hypersonic speed for a blunt body, based on numerical simulation, including the pressure distribution near the side jet nozzle and the effect of the angle of attack on the side jet interaction with the blunt body. Characteristics of skin friction patterns, at different angles of attack, obtained from numerical simulation and thier associated flow fields are presented in this paper.

\section{Flow Conditions}

The same blunt body model as investigated by $\mathrm{Ku}$ rita et al. ${ }^{10)}$ is considered in the present study, which is a $10^{\circ}$ half-angle blunted cone with a circular sonic jet nozzle installed flush-mounted on the body surface. A schematic of the model employed in this study is shown in Fig. 1 along with the coordinate system.

The freestream and jet conditions are summarized in Tables 1 and 2, respectively. The ratio of the jet static pressure $p_{j}$ to the uniform flow static pressure $p_{\infty}$, which is referred to as the nozzle pressure ratio $(\mathrm{NPR})$, is $\mathrm{NPR}=p_{j} / p_{\infty}=1.01 \times 10^{3}$.

Table 1. Freestream conditions.

\begin{tabular}{ll}
\hline \hline Parameter & Value \\
\hline Mach number & $M_{\infty}=8.1$ \\
Attack angle & $\alpha=0.0^{\circ}, 20.0^{\circ}, 40.0^{\circ}$ \\
Total pressure & $P_{o}=4.00 \mathrm{MPa}$ \\
Total temperature & $T_{o}=900 \mathrm{~K}$ \\
Pressure & $p_{\infty}=371 \mathrm{~Pa}$ \\
Temperature & $T_{\infty}=63.7 \mathrm{~K}$ \\
Density & $\rho_{\infty}=0.0203 \mathrm{~kg} / \mathrm{m}^{3}$ \\
Reynolds number & $\operatorname{Re}_{L}=7.5 \times 10^{5}$ \\
Working fluid & $\mathrm{Air}$ \\
\hline \hline
\end{tabular}

The mass flow ratio of the jet to the freestream, $\dot{m}$, is defined by:

$$
\dot{m}=\frac{\rho_{j} V_{j} A_{j}}{\rho_{\infty} V_{\infty} A_{b}}=\left(\frac{p_{j} M_{j} A_{j}}{p_{\infty} M_{\infty} A_{b}}\right)\left(\frac{\gamma_{j} R_{\infty} T_{\infty}}{\gamma_{\infty} R_{j} T_{j}}\right)^{\frac{1}{2}}
$$

In this study, $\dot{m}=5.34 \times 10^{-2}$. In addition, the momentum flux ratio, $\phi_{j} / \phi_{\infty}$, is defined by:

$$
\frac{\phi_{j}}{\phi_{\infty}}=\frac{\rho_{j} V_{j}^{2} A_{j}}{\rho_{\infty} V_{\infty}^{2} A_{b}}=\frac{\gamma p_{j} M_{j}^{2} A_{j}}{\gamma p_{\infty} M_{\infty}^{2} A_{b}}
$$

In this study, $\phi_{j} / \phi_{\infty}=1.31 \times 10^{-2}$.

Table 2. Jet conditions.

\begin{tabular}{ll}
\hline \hline Parameter & Value \\
\hline Mach number & $M_{j}=1.0$ \\
Total pressure & $P_{o j}=0.71 \mathrm{MPa}$ \\
Total temperature & $T_{o j}=288 \mathrm{~K}$ \\
Pressure & $p_{j}=0.373 \mathrm{MPa}$ \\
Temperature & $T_{j}=240 \mathrm{~K}$ \\
Density & $\rho_{j}=5.41 \mathrm{~kg} / \mathrm{m}^{3}$ \\
Working fluid & $\mathrm{Air}$ \\
\hline \hline
\end{tabular}

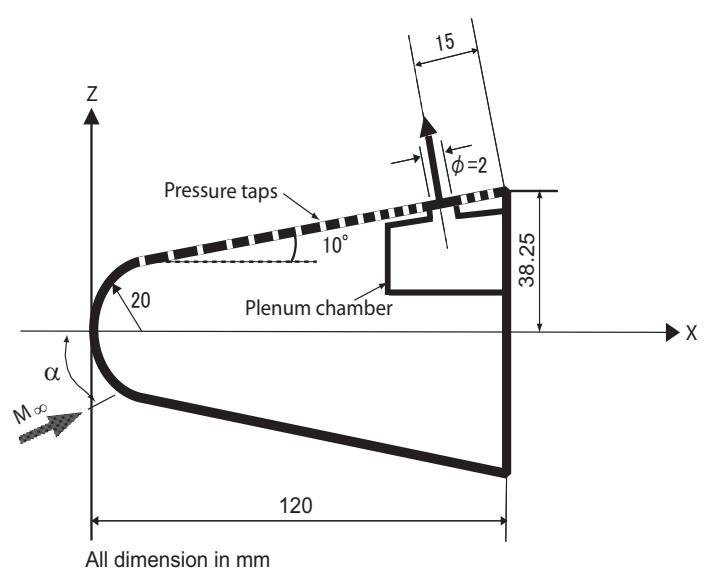

Fig. 1. Model and coordinate system used by Kurita et al. ${ }^{10)}$ 


\section{Numerical Method and Grid System}

An explicit Navier-Stokes solver based on the finite volume formulation was employed for the present flow simulation. The inviscd numerical fluxes are obtained by AUSM ${ }^{13)}$ along with the third-order monotonic upwind scheme for conservation law (MUSCL). The viscous terms are approximated by centralized differentiation, and time integration is carried out by the second-order Runge-Kutta scheme. Computations were performed by the Nagoya University supercomputer (Fujitsu PRIMEPOWER HPC2500) using 10 CPUs.

The computational grid system employed in the present study consists of two blocks. The first block is for the three-dimensional model alone. The number of grid points along the body surface is 135 points in the streamwise direction, where the minimum grid spacing is $1.0 \times 10^{-4}$. The number of grid points in other directions is 50 points for each direction with the same minimum grid spacing as that in the streamwise direction. Thus, the total number of grid points for the first block system is 337,500 . The first block extends $1.2 \mathrm{~L}$ along the negative $\mathrm{x}$-axis and $1.6 \mathrm{~L}$ in the radial direction.

The second block is for the base and wake regions. This block consists of 50 points in the streamwise direction, 100 points in the radial direction, and 50 points in the circumferential direction. The second block extends $1.6 \mathrm{~L}$ in the radial direction and $1.7 \mathrm{~L}$ along the positive $\mathrm{x}$-axis. Thus, the total number of grid points for these two blocks is 587,500 , and the grid system is shown in Fig. 2.

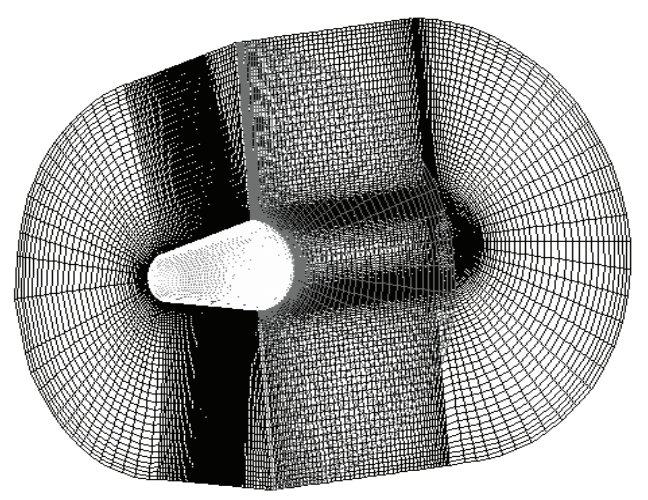

Fig. 2. Grid system used in the present study (587,500 grid points).

\section{Results}

\subsection{Effects of attack angle on flow field}

\subsubsection{The case of $\alpha=0^{\circ}$}

Figures 3(a) and 3(b) show the overall schlieren visualizations in experiments and $\mathrm{CFD}$, respectively, for the case of $\alpha=0^{\circ}$. The computed flow pattern captures several flow characteristics such as the jet bow shock and the separation shock which were caused by the side jet emitted from the body surface.

A close-up of velocity vectors in the jet flow and its interaction with neighboring flows and pressure distribution in the symmetric plane as well as the surface flow pattern are shown in Fig. 4(a), where its schematics for flow features are illustrated in Fig. 4(b). The surface pressure upstream of the jet increases due to the jet bow shock (JBS), so that the adverse pressure gradient is produced there and at the wall, which leads to boundary layer separation (SBL) and separation shock (JSC).

The jet bow shock acts as a wall to the incoming flow, which extends laterally around the jet. From the velocity vectors shown in Fig. 4(a), three vortices can be observed. Upstream of the jet, a clockwise separation vortex (V1) and a counterclockwise horseshoe vortex (V2) exist, while downstream of the jet, a clockwise recompression vortex (V3) exists. Their significance on the interacting flow fields will be discussed later in detail.

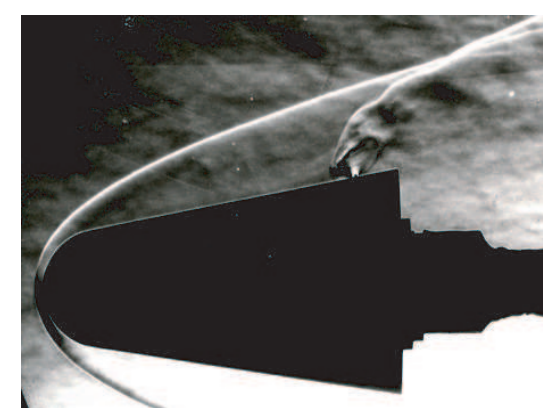

(a) Experiment

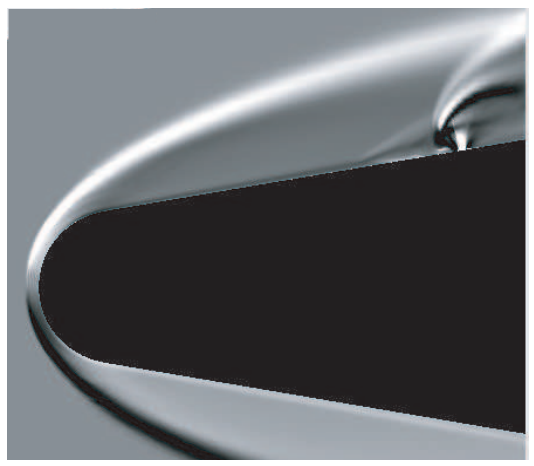

(b) CFD

Fig. 3. Comparison of schlieren visualization between experiment ${ }^{10}$ ) and computation at $\alpha=0^{\circ}$. 
The pressure distribution along the body surface on the leeward side in the symmetry plane is shown in Fig. 5. The marks represent experimental data, and the lines for numerical results; the solid line is for jet-on case and the dotted line for jet-off case. The point at $x / L=0$ corresponds to the jet center.

Both the experimental and numerical results are in reasonable agreement. In the jet-on case, the first increase in pressure occurs at about $x / L \approx-0.28$. This is caused by the separation shock due to the boundary layer separation. Another high-pressure point is seen at $x / L \approx-0.06$ with $p / p_{\infty}=21.0$ due to the jet bow shock, where a flow reversal occurs near the wall as shown in Fig. 4.

Figure 6 shows the computed surface flow patterns and pressure distribution on the body surface for jet-on and jet-off cases. The flow is attached everywhere in the jet-off case as shown in Fig. 6(a). All skin friction lines originate at the nodal point of attachment at the nose.

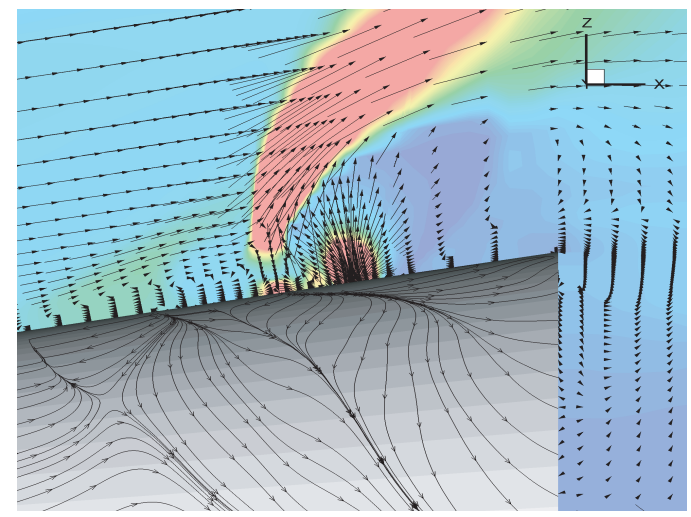

(a) Velocity vectors and pressure field

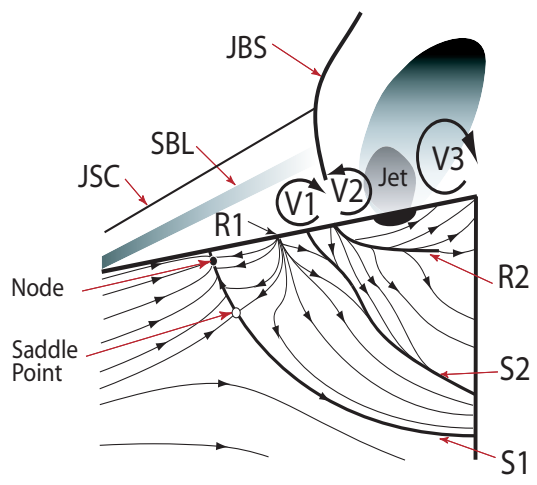

(b) Schematic

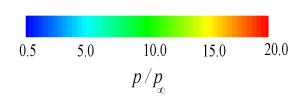

Fig. 4. Close-up of jet flow field in the symmetric plane and on body surface $\left(\alpha=0^{\circ}\right)$ : JBS, jet bow shock; JSC, jet separation shock ; S1, S2, separation lines; R1, R2, reattachment lines; SBL, separated boundary layer; V1, separation vortex; V2, horseshoe vortex; V3, recompression vortex; $\circ$, saddle point; $\bullet$, node point.
Figure 6(c) represents the schematic of the surface flow pattern shown in Fig. 6(b) for the jet-on case. The jet injection dramatically alters the topological structure of the pattern of skin friction lines on the leeward side near the base. The flow separation occurs upstream of the jet on the leeward side along the separation line $(\mathrm{S} 1)$, which is located at $x / L \approx-0.25$ on the symmetry plane and extends to the base at a meridian angle of $\theta \approx \pm 90^{\circ}$. Three saddle points are observed on this separation line (S1), which identifies it as global flow separation line. ${ }^{12)}$ A region of reverse flow exists downstream of this separation line $(\mathrm{S} 1)$ on the leeward side and is bounded by the reattachment line $(\mathrm{R} 1)$ at $x / L \approx-0.13$ on the symmetry plane and extends back to $x / L \approx-0.11, \theta \approx \pm 15^{\circ}$. The increase in pressure at $x / L \approx-0.13$ shown in Fig. 5 is attributed to flow reattachment at this point.

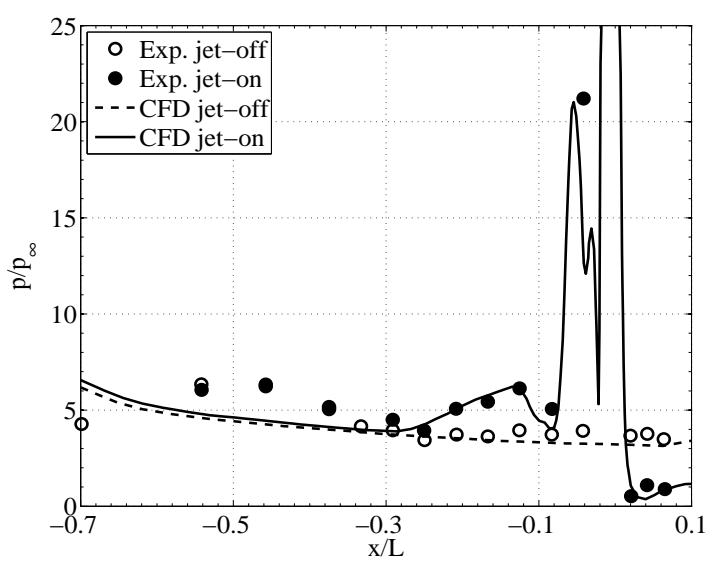

Fig. 5. Pressure distribution along the body surface on the leeward side $\left(\alpha=0^{\circ}\right)$.

The secondary flow separation line (S2) occurs on the leeward side downstream of the reattachment line (R1) at $x / L \approx-0.11$ on the symmetry plane back to the base at $\theta \approx \pm 85^{\circ}$. The flow separation due to the separation vortex (V1), shown in Fig. 4(b), is contributed to the occurrence of the secondary separation line (S2). Flow reattaches again on the leeward side at $x / L \approx-0.05$ on the symmetry plane and extends back to $x / L \approx+0.025, \theta \approx \pm 30^{\circ}$ along the reattachment line $(\mathrm{R} 2)$. The flow reattaches due to the flow circulation from the separation vortex (V1) and horseshoe vortex (V2), shown in Fig. 4(b). These two vortices decrease the surface pressure, which corresponds to at $x / L=-0.08$ and $x / L=-0.021$ in Fig. 5. These trends in pressure computations show reasonable agreement with the experiment. Discrepancy between experiment and computation occurs around $x / L=-0.5$ upstream of the boundary layer separation in Fig. 5. The reason behind these discrepancies is not clear from the present computations. Downstream of the jet and near the base, a recom- 
pression vortex (V3) exists. The low-pressure region upstream of the reverse flow region on the leeward side, shown in Fig 6(b), creates a favorable circumferential pressure gradient that drives the skin friction lines leeward with jet injection as shown in Figs. 4 and $6(\mathrm{c})$.

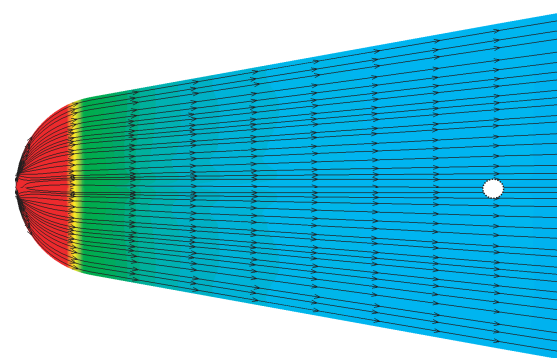

(a) Jet-off

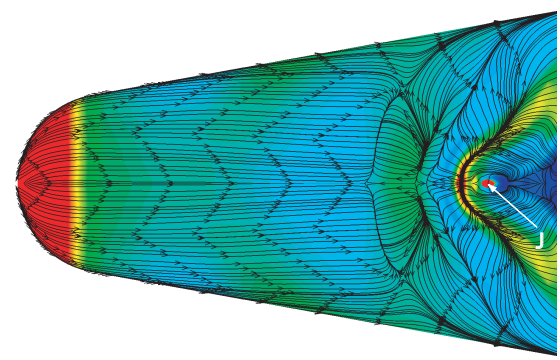

(b) Jet-on

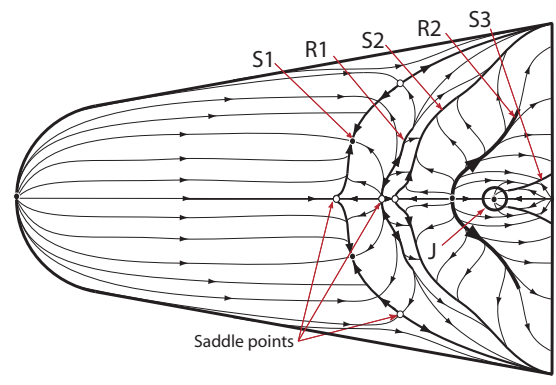

(c) Schematic (jet-on)

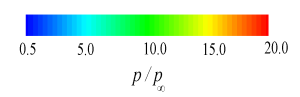

Fig. 6. Computed upper-surface flow patterns and pressure distribution at $\alpha=0^{\circ}$ : J, jet; S1, S2, S3, separation lines; R1, R2, reattachment lines; o, saddle points; •, node points.

It should be noted that, such surface flow patterns can be produced in wind tunnels using the oil flow visualization technique if the running time of the tunnel is long enough. However, the present shock tunnel has a running time of less than 100 milliseconds, which makes the oil flow visualization technique difficult to apply in these experiments. ${ }^{14)}$ Therefore, the present computations are indispensable to understand the jet flow field interaction with the available limited experimental results.

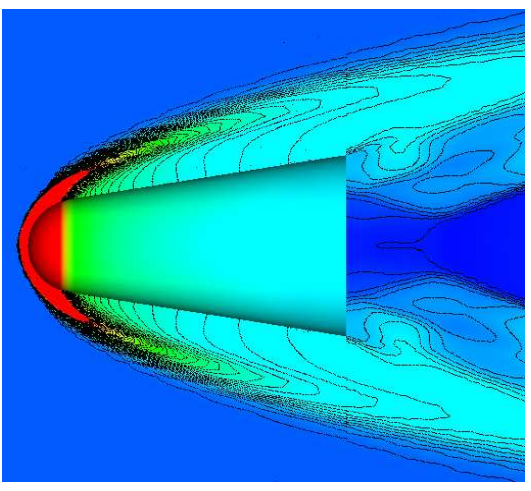

(a) Jet-off

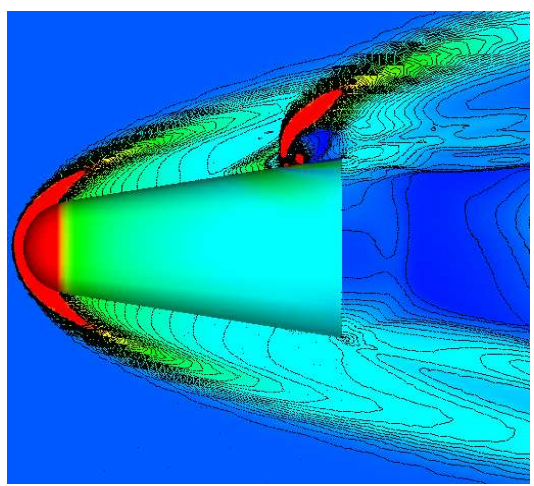

(b) Jet-on

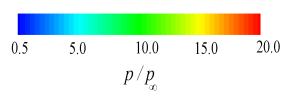

Fig. 7. Pressure contours on the symmetric plane and body surface for jet-on and jet-off cases $\left(\alpha=0^{\circ}\right)$.

Incidentally, there is a large difference between the jet-on and jet-off cases in the wake flow pattern; the low-pressure region in the jet-on case extends more in the lateral direction compared with that in the jet-off case, as shown in Fig. 7. In experiments, the effect of side jet on the wake structure cannot be studied due to the effect of a supporting sting.

\subsubsection{The case of $\alpha=20^{\circ}$}

Figures 8(a) and 8(b) show experimental and computational schlieren visualizations, respectively, for the case of $\alpha=20^{\circ}$. Reasonable agreement between them is obtained. The main bow shock, the jet bow shock and the jet boundary are captured by computations and are in reasonable agreement with the experimental results shown in Fig. 8(a). The separation shock is clearly shown as a dark thick line at the leeward side near the surface in Fig. 8(b). Comparing the results of $\alpha=0^{\circ}$ and $20^{\circ}$, it is found that the separation shock is located closer to the nose in the case of $\alpha=20^{\circ}$ than $\alpha=0^{\circ}$, which agrees with the experimental observation. In addition, the jet boundary expands larger than the case of $\alpha=0^{\circ}$, as shown in Fig. 3. 


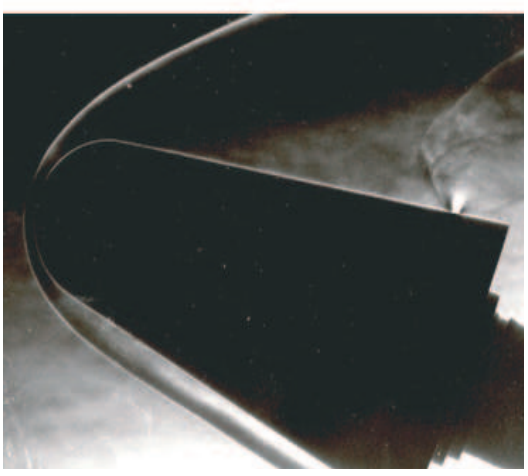

(a) Experiment

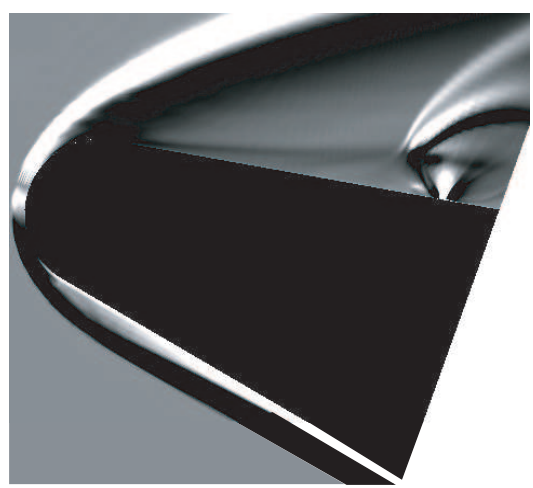

(b) CFD

Fig. 8. Comparison of schlieren visualization between the experiment ${ }^{10)}$ and computation at $\alpha=20^{\circ}$.

The pressure distributions along the body surface on the leeward side in the symmetry plane are shown in Fig. 9. Note that the vertical scale is different between $\alpha=0^{\circ}$ and $\alpha=20^{\circ}$, where the pressure has been reduced on the leeward side in the case of $\alpha=20^{\circ}$. The general tendency is similar in both cases. Since the jet becomes stronger relative to the ambient pressure in the case of $\alpha=20^{\circ}$, the pressure increase leading to boundary layer separation propagates further upstream. Figure 10 shows the computed surface flow patterns and pressure distribution on the body surface for jet-on and jet-off cases. The surface flow patterns are also depicted in the schematics shown in this figure. At this angle of attack without the jet injection, as shown in Fig. 10(a,b), the topological structure of the pattern of skin friction lines is considerably altered compared with the similar case of $\alpha=0^{\circ}$. All skin friction lines again originate at the nodal point of attachment. The crossflow caused by incident is clearly shown. The angle $\delta_{s}$ that the skin-friction lines greet the body axis was measured along the sides at $\theta \approx \pm 90^{\circ}$. Near the nose, $x / L \approx-0.85, \delta_{s}=22^{\circ}$, which is about 0.5 the potential angle ${ }^{15)}$ at the edge of the boundary layer of $\delta_{e}=2 \alpha=40^{\circ}$. Rearward of $x / L \approx-0.85$, the sur- face flow angle increases with increasing length until $\delta_{s}=45^{\circ}$ at the base, which is close to $\delta_{e}$. The significantly lower flow angles near the nose mean that local flow velocity distribution is skewed in the direction aft of the inviscid streamline. It should be noted that the favorable crossflow pressure gradient is responsible for the increase in flow angle as shown from the pressure distribution on the body surface presented in Fig. 10(a).

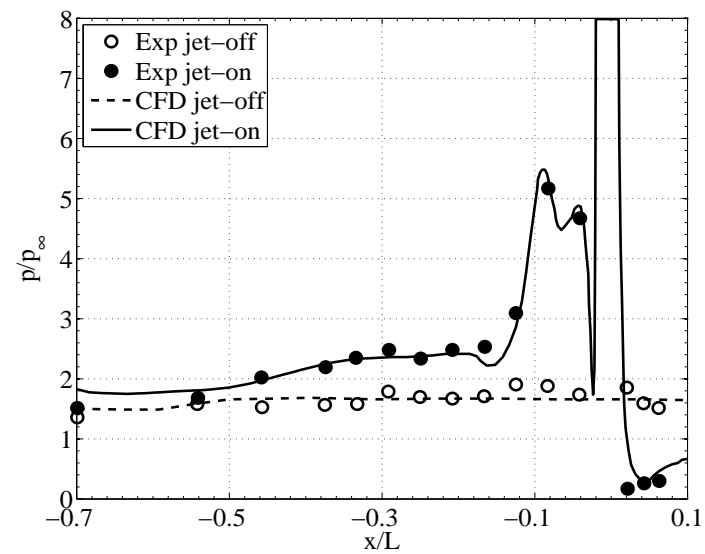

Fig. 9. Pressure distribution along the body surface on the leeward side $\left(\alpha=20^{\circ}\right)$.

The skin friction lines at the leeward side are attached and axially directed all the way until $x / L \approx$ -0.15 . A round nose and favorable pressure gradient can be attributed for the existence of strong axial flow, which contributed to the delay in flow separation. However, according to conical flow concepts, local separation at the nose was expected for $\alpha=20^{\circ}>\delta_{b}$, where $\delta_{b}$ is the blunted cone half-angle of $10^{\circ}$ for the present blunt body. The flow separation occurs on the leeward side at the separation line (S) that occurs at $x / L \approx-0.25, \theta \approx \pm 22^{\circ}$ back to base at $\theta \approx \pm 53^{\circ}$. Emanating from a node rather than a saddle point, this particular line qualifies as a local line of separation. ${ }^{12)}$

The computed surface flow patterns and pressure distribution on the body surface for jet-on are shown in Figure 10(c). The surface flow pattern schematic is shown in Fig. 10(d). The jet injection dramatically alters the topological structure of the pattern of skin friction lines on the leeward side near the base compared to the jet-off case. The topological structure is similar to the corresponding case of $\alpha=0^{\circ}$. The flow separation occurs upstream of the jet on the leeward side at the separation line (S1) located at $x / L \approx-0.32, \theta \approx \pm 7^{\circ}$ and extends to the base at $\theta \approx \pm 65^{\circ}$, which propagates further upstream compared with the corresponding case of $\alpha=0^{\circ}$. 
M. K. IBRAHIM et al.: The Role of Vortices in Side Jet/Blunt Body Interaction at Hypersonic Speed

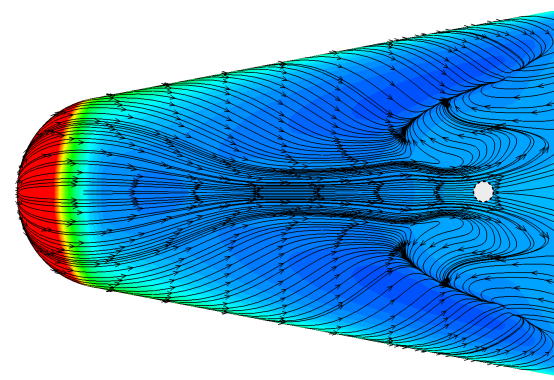

(a) Jet-off

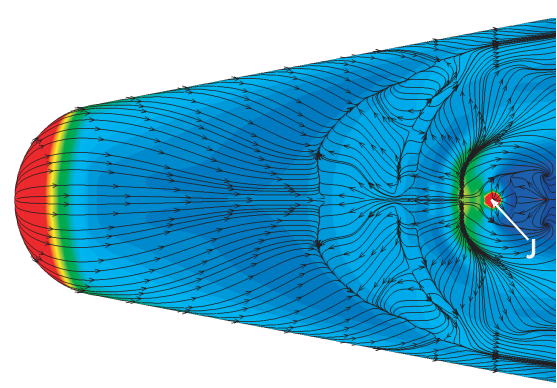

(c) Jet-on

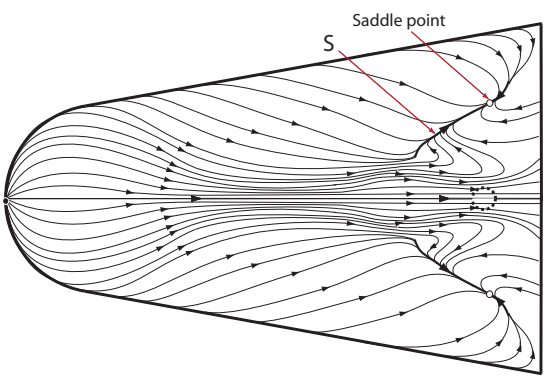

(b) Schematic (jet-off)

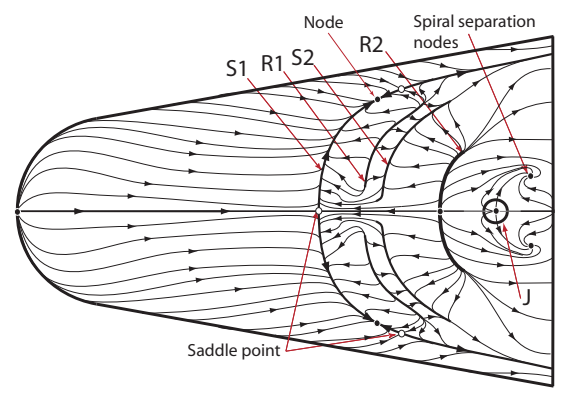

(d) Schematic (jet-on)

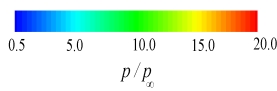

Fig. 10. Computed upper-surface flow patterns and pressure distribution at $\alpha=20^{\circ}$ : J, jet; S, S1, S2, separation lines; R1, R2, reattachment lines; o, saddle points; •, node points.

Three saddle points are observed on this separation line (S1), which identifies it as a global flow separation line. ${ }^{12)}$ The region of reverse flow exists downstream of this separation line $(\mathrm{S} 1)$ on the leeward side and is bounded by the reattachment line (R1) at $x / L \approx-0.18$ on the symmetry plane and extends downstream to $x / L \approx-0.1, \theta \approx \pm 39^{\circ}$. It should be noted that the region of reverse flow on the symmetry plane extends further downstream to $x / L \approx-0.06$. The slight increase in pressure around $x / L \approx-0.18$ shown in Fig. 9 is attributed to flow reattachement at this point.

The flow separation due to the separation vortex is contributed to the occurrence of the secondary separation line (S2). This line occurs on the leeward side downstream of the reattachment line (R1) at $x / L \approx-0.14$ on the symmetry plane and extends back to $x / L \approx-0.09, \theta \approx \pm 39^{\circ}$. Flow reattaches again on the leeward side at $x / L \approx-0.06$ on the symmetry plane and extends back to $x / L \approx+0.04$, $\theta \approx \pm 17^{\circ}$ along the reattachment line (R2). The flow reattaches due to the flow circulation from the separation vortex and horseshoe vortex. These two vortices decrease the surface pressure, which corresponds to $x / L \approx-0.15$ and $x / L \approx-0.02$, shown in Fig. 9. The increase in pressure around $x / L \approx-0.06$ in Fig. 9 is attributed to the jet bow shock wave. These trends in pressure computations show reasonable agreement with the experiment. Downstream of the jet and at $\theta \approx \pm 8^{\circ}$, two spiral separation nodes can be observed as shown in Fig. 10(c, d).

Since the main flow has rather low pressure on the leeward side for $\alpha=20^{\circ}$ due to the separation vortices, the jet flow expands larger than the case of $\alpha=0^{\circ}$. Passing through the jet bow shock wave, the flow goes toward the surface. After impinging the surface, most of the flow changes direction toward the jet, because the pressure in the expanding jet flow is low. Less reversed flow appears in the case $\alpha=20^{\circ}$, resulting in a weak separation vortex compared with the case of $\alpha=0^{\circ}$. On the other hand, the separation and horseshoe vortices still exist and affect the pressure distribution, as in the case of $\alpha=0^{\circ}$.

\subsubsection{The case of $\alpha=40^{\circ}$}

Similarly, Figs. 11(a) and 11(b) show experimental and computational schlieren visualizations, respectively, for the case of $\alpha=40^{\circ}$. Both the blunt body bow shock and weak jet bow shock are captured in computations with reasonable agreement with the experiments. The computed pressure field (not presented here) shows that there are also weak shock waves caused by boundary layer separation. Com- 
pared with the lower attack angle cases, the locations moves upstream.

The pressure distributions along the body surface on the leeward side in the symmetry plane are shown in Fig. 12. The pressure increase at $x / L=-0.04$ is attributed to the jet bow shock wave. The tendency is slightly different from the previous two cases. As shown before, the clockwise vortex does not exist in this case. Moreover, the influence of the counterclockwise vortex on the surface pressure becomes weak. However, a massive separated flow region, which is not observed in the plane of symmetry of the model, still exists upstream of the jet.

Figure 13 shows the computed surface flow patterns and pressure distribution on the body surface for jet-on and jet-off cases. The surface flow patterns are also depicted in the schematics shown in this figure. At this angle of attack, without the jet injection as shown in Fig. 13(a,b), the topological structure of the pattern of skin friction lines is considerably altered compared with similar cases of $\alpha=0^{\circ}$ and $\alpha=20^{\circ}$. Skin friction lines again originate at the nodal point of attachment.

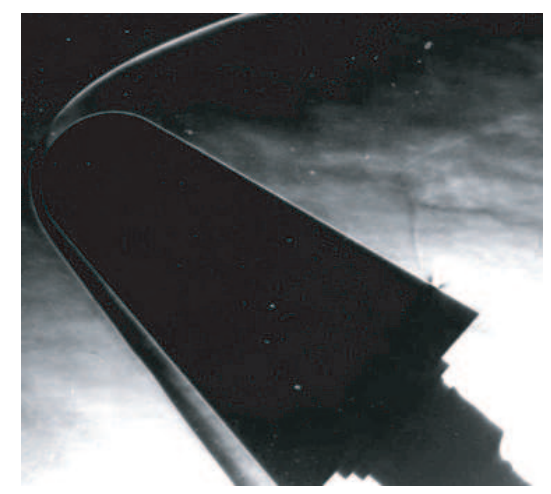

(a) Experiment

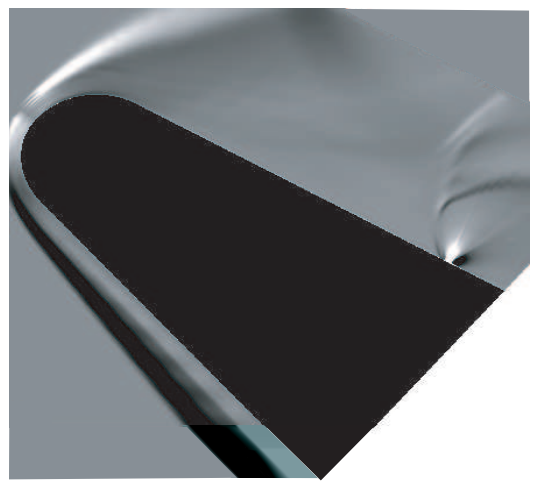

(b) $\mathrm{CFD}$

Fig. 11. Comparison of schlieren visualization between the experiment ${ }^{10)}$ and computation $\left(\alpha=40^{\circ}\right)$.

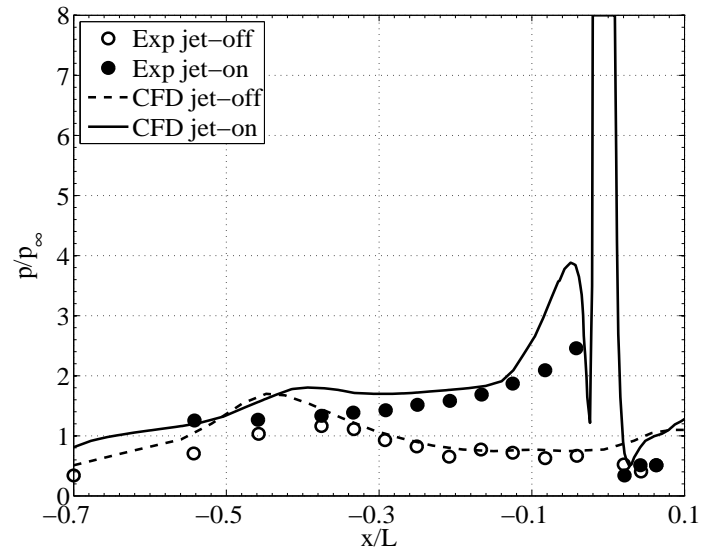

Fig. 12. Pressure distribution along the body surface on the leeward side $\left(\alpha=40^{\circ}\right)$.

The crossflow caused by the incident is clearly shown. Global flow separation occurs on the leeward side along the separation line (S1) that occurs on the symmetry plane and $x / L \approx-0.68$ back to $x / L \approx+0.02$, $\theta \approx \pm 40^{\circ}$. A small reverse flow bubble is formed on the leeward side between $-0.68 \leq x / L \leq-0.56$. The secondary separation ( $\mathrm{S} 2)$ line that occurs at $\theta \approx$ $\pm 12^{\circ}$ and extends between $-0.44 \leq x / L \leq-0.13$, can be observed. In this type of flow pattern, the flow from the primary separation vortex reattaches at $\theta=0^{\circ}$ and moves toward $\theta \approx \pm 12^{\circ}$, where it locally separates again into a local vortex filament that circulates in the opposite direction to the primary vortex. This vortex filament locally reattaches along the reattachment line $(\mathrm{R} 1)$ at $\theta \approx \pm 20^{\circ}$. A large part of this primary reattachment vortex flow moves in downstream direction. Another reattachment line (R2) at $x / L \approx 0.00$ and $\theta \approx \pm 20^{\circ}$ can be observed.

The computed surface flow patterns and pressure distribution on the body surface for jet-on are shown in Fig. 13(c). The surface flow pattern schematic is shown in Fig. 13(d). The jet injection alters the topological structure of the pattern of skin friction lines on the leeward side compared to the jet-off case. Global flow separation occurs on the leeward side along the separation line $(\mathrm{S} 1)$, which is located at $x / L \approx-0.66$ on the symmetry plane and extends to the base at $\theta \approx \pm 42^{\circ}$. Similar to the jet-off case, a small reverse flow bubble is formed on the leeward side between $-0.66 \leq x / L \leq-0.54$. The flow from the primary separation vortex reattaches at $\theta=0^{\circ}$ and moves toward the separation line (S1). A large part of this reattached flow moves in the downstream direction, where it bypasses the jet and separates along the separation line (S2). Reverse flow downstream of the jet can be observed. There are neither secondary separation lines nor reattachment lines observed with the jet-on case. From the velocity vector distribution at $x / L=0$ plane (not presented here) for the two cases: 


\section{K. IBRAHIM et al.: The Role of Vortices in Side Jet/Blunt Body Interaction at Hypersonic Speed}

$\alpha=20^{\circ}$ and $40^{\circ}$, it is found that the jet expands larger at $\alpha=40^{\circ}$. Due to the high-pressure region caused by the jet bow shock, the flow is blocked by the jet in the case of $\alpha=20^{\circ}$, creating a vortex. This vortex also decreases the surface pressure. This vortex seems to be weakened in the $\alpha=40^{\circ}$ case.

\subsection{Jet amplification factor}

The jet amplification factor can be defined as follows:

$$
K=\frac{\Upsilon_{V A}+\Delta F_{i}}{\Upsilon_{V A}}=1+\frac{\Delta F_{i}}{\Upsilon_{V A}}
$$

where $\Delta F_{i}$ is the force produced by the interaction and $\Upsilon_{V A}$ is the reaction force by the jet injected into a vacuum.

$$
\Delta F_{i}=F_{J-O N}-F_{J-O F F}=\sum(\Delta X \times \Delta p)
$$

The jet thrust is obtained by assuming that the jet injects into a vacuum as an isentropic flow as follows:

$$
\Upsilon_{V A}=\dot{m}_{j} V_{j}+p_{j} A_{j}
$$

The amplification due to the side jet aerodynamic interaction is composed of two parts: the extent of an interaction region and the magnitude of pressure increase. When there is no aerodynamic interaction force, the amplification factor becomes unity. This factor usually attains the value of more than two in two-dimensional cases, ${ }^{3}$ ) while in three dimensional case it becomes lower than that ${ }^{2,4,16,17)}$.

Table 3. Jet amplification factor.

\begin{tabular}{llll}
\hline \hline$\alpha$ & CFD & Experiment $^{10)}$ & Error [\%] \\
\hline $0^{\circ}$ & 1.368 & 1.367 & 0.07 \\
$20^{\circ}$ & 1.432 & 1.438 & 0.42 \\
$40^{\circ}$ & 1.338 & 1.375 & 2.77 \\
\hline \hline
\end{tabular}

The results of the jet amplification factor are shown in Table 3. Both computational and experimental results are in good agreement. These results indicate that the interaction enhances the jet reaction forces, where about $35 \%$ - $45 \%$ of the jet reaction force acts on the surface due to the interaction. Thus, this influence cannot be neglected.

\section{Conclusions}

Numerical simulations were carried out to explore the aerodynamic interaction between a hypersonic flow and a sonic side jet emitted normally from the surface of the leeward side of a blunt body. The numerical simulation showed reasonable agreement with experimental data in terms of schlieren visualization and surface pressure measurement. Based upon the results presented above, the following conclusions can be drawn:

1. At low- and middle-angles of attack, it is made clear that both the separation vortex and horseshoe vortex are formed inside the separated boundary layer upstream of the side jet. Their presence was reflected in the measured surface pressure, and it was made clear that they are responsible for the drops observed in the pressure distributions.

2. High angle of attack results showed that the role of vortices have little influence on the pressure distribution and the interaction of the jet with external flow produces a complex flow field that may be difficult to characterize using a simpler approach. ${ }^{7)}$

3. The jet force amplification results indicate that the interaction enhances the jet reaction forces, where $35 \%-45 \%$ of the jet reaction force acts on the surface due to the interaction. Thus, this influence cannot be neglected. These results depend on both the high pressure body surface area and the jet strength and show nonlinear characteristics with angle of attack.

\section{References}

1) Graham, M. J. and Weinacht, P.: Numerical Simulation of Lateral Control Jets, AIAA Paper 99-0510, 1999.

2) Kontis, K. and Stolley, J. L.: Control Effectiveness of a Jet Slender Body Combination at Hypersonic Speeds, Journal of Spacecraft and Rockets, 34(1997), pp. 762-768.

3) Frank, W. S.: Two-Dimentional Jet Interaction Studies at Large Values of Reynolds and Mach Numbers, AIAA Journal, 13(1975), pp. 1430-1434.

4) Julius, B. and Jacob, G.: Experimental Investigation of Side Jet Steering for Supersonic and Hypersonic Missiles, Journal of Spacecraft and Rockets, 33(1996), pp. 346-352.

5) Goldman, R. L.: Unsteady Control Surface Loads of Lifting Re-entry Vehicles at Very High Speeds, AIAA Journal, 6(1968), pp. 44-50.

6) Joseph, A. S., Paul, F. H. and Harry, L.: Structure of Highly Underexpanded Transverse Jets in a Supersonic Stream, AIAA Journal, 5(1967), pp. 882-884.

7) Jurgeb, T.: Semi-Emprical Prediction of Lateral Control Jet Flow Field Feature in Hypersonic Flow, AIAA Paper 99-0805, 1999.

8) Kurita, M., Okada, T. and Nakamura, Y.: The Effects of Attach Angle on Aerodynamic Interaction due to Side Jet from a Blunted Body in a Supersonic Flow, AIAA Paper 2001-0261, 39th AIAA Aerospace Sciences Meeting and Exhibit 8th-11th January 2001 Reno, NV. 


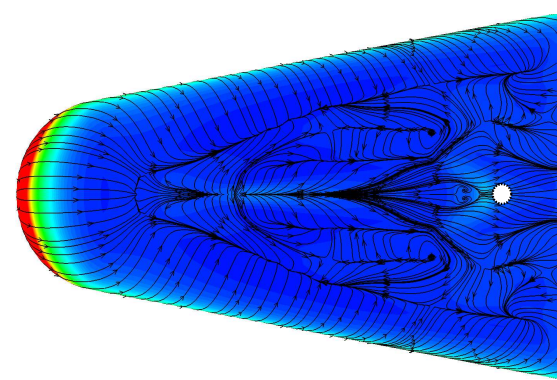

(a) Jet-off

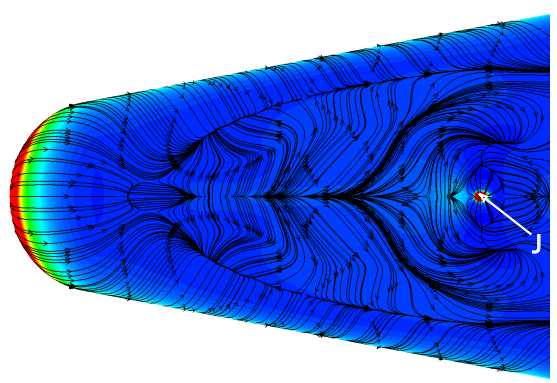

(c) Jet-on

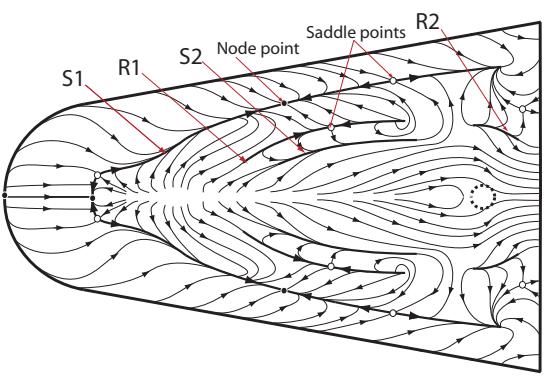

(b) Schematic (jet-off)

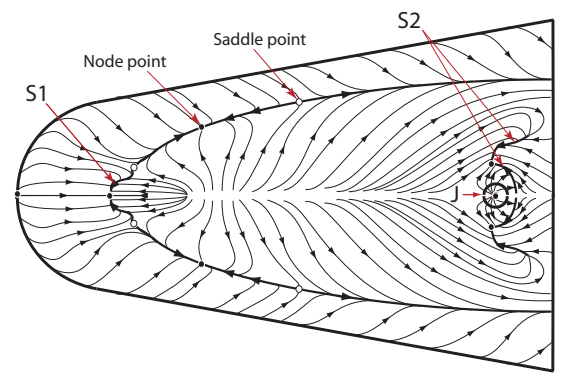

(d) Schematic (jet-on)

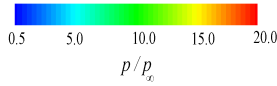

Fig. 13. Computed upper-surface flow patterns and pressure distribution at $\alpha=40^{\circ}$ : J, jet; S1, S2, separation lines; R1, R2, reattachment lines; o, saddle points; •, node points.

9) Kurita, M., Okada, T., Nakamura, Y., Hongo, M. and Inatani, Y.: Effects of Attack Angle on Side Jet Interaction in Supersonic Flow, Journal of the Japan Society for Aeronautical and Space Sciences, 50(2002), pp. 310-317, (in Japanese).

10) Kurita, M., Okada, T. and Nakamura, Y.: Side Jet Aerodynamic Interaction on a Blunt Body in Hypersonic Flow, Journal of the Japan Society for Aeronautical and Space Sciences, 50(2002), pp. 394-401, (in Japanese).

11) Kurita, M., Okada, T. and Nakamura, Y.: PressureSensitive Paints Measurement of Hypersonic Flow in Shock Tunnel, Journal of the Japan Society for Aeronautical and Space Sciences, 51(2003), pp. 10-16, (in Japanese).

12) Peake, D. J. and Tobak, M.: Three-Dimensional Separation and Reattachment, NASA Technical Memorandum 84221, 1982.
13) Liou, M.-S. and Steffen, C. J.: A New Flux Splitting Scheme, Journal of Computational Physics, 107(1993), pp. 23-39.

14) Kurita, M., Okada, T. and Nakamura, Y.: The Effects of Attach Angle on Side Jet Aerodynamic Interaction in a Blunted Body at Hypersonic Flow, AIAA Paper 20011825, AIAA/NAL-NASDA-ISAS 10th International Space Planes and Hypersonic Systems and Technologies Conference, 24-27 April, 2001, Kyoto, Japan.

15) Keener, E. R.: Oil Flow Separation Patterns on an Ogive Forebody, AIAA Journal, 21(1983), pp. 550-556.

16) Kennedy, K., Walker, B. and Mikkelsen, C.: Jet Interaction Effects on Missile with Aerodynamic Control Surface, AIAA Paper 90-0807, 1990.

17) Shang, J. S.: Interaction of Jet in Hypersonic Cross Stream, AIAA Journal, 27(1989), pp. 323-329. 